\title{
The GO4 Model in Near-Nadir Microwave Scattering From the Sea Surface
}

\author{
Boisot Olivier ${ }^{1,2}{ }_{-}^{*}$, Nouguier Frederic ${ }^{1}$, Chapron Bertrand ${ }^{3}$, Guerin Charles-Antoine ${ }^{1}$
}

1 Aix Marseille Univ, Univ Toulon, CNRS, MIO,IRD,UM 110, F-83957 La Garde, France.
2 Ctr Natl Etud Spatiales, Collecte Localisat Satellites, F-31400 Toulouse, France.
${ }^{3}$ IFREMER, Lab Oceanog Spatiale, F-29280 Plouzane, France.

Corresponding author : Olivier Boisot, email address : olivier.boisot@univ-tln.fr ;

frederic.nouguier@univ-tln.fr ; bertrand.chapron@ifremer.fr ; querin@univ-tln.fr

\begin{abstract}
:
We introduce a practical and accurate model, referred to as "GO4," to describe near-nadir microwave scattering from the sea surface, and at the same time, we address the issue of the filtered mean square slope (mss) conventionally used in the geometrical optics model. GO4 is a simple correction of this last model, taking into account the diffraction correction induced by the rough surface through what we call an effective mean square curvature (msc). We evaluate the effective msc as a function of the surface wavenumber spectrum and the radar frequency and show that GO4 reaches the same accuracy as the physical optics model in a wide range of incidence and frequency bands with the sole knowledge of the mss and msc parameters. The key point is that the mss entering in GO4 is not the filtered but the total slope. We provide estimation of the effective msc on the basis of classical sea spectrum models. We also evaluate the effective msc from near-nadir satellite data in various bands and show that it is consistent with model predictions. Non-Gaussian effects are discussed and shown to be incorporated in the effective msc. We give some applications of the method, namely, the estimation of the total sea surface mss and the recalibration of relative radar cross sections.
\end{abstract}

Keywords : Curvature, geometrical optics, near-nadir, ocean radar sensing, slope 


\section{INTRODUCTION}

In spite of more than one half-century of theoretical developments in backscattering from the sea surface (e.g. [1]), the ever increasing capabilities of spaceborne microwave sensors still triggers the need for accurate, simple and versatile models for the geophysical interpretation of multi-frequency active and passive microwave data sets. Starting from the historical asymptotic theories which have a limited domain of application, many robust analytical scattering models have been developed in the last three decades [2]. Some of them have proven to be particularly relevant for the ocean surface (e.g. [3], [4], [5], [6], [7], [8]), with a wide range of validity in terms of incidence angles, radar frequency and sea state. Now, virtually all these so-called unified models rely on the assumed knowledge of the sea surface wave number spectrum which is difficult to use in operational conditions, both from a conceptual and technical point of view. For this reason the most practical method at low incidence remains the historical Geometrical Optics (which we abbreviate to "GO2" to distinguish it from GO4) approach which relates the backscattered power to the mere probability distribution of surface slopes and is usually parametrized by the sole mean square slope (mss). However, as it is well known, this asymptotic theory is only valid in the optical limit of very short radar wavelength and can deviate significantly from the actual backscattering cross section in the microwave regime where it fails to reproduce the radar sensitivity to radar wavelength. It is often resorted to a "radar-filtered" mss ([9], [10], [11], [12], [13]) which, as we will see, is an artificial compensation of the missing diffraction term in $\mathrm{GO} 2$ and accounts for the fact that roughness scales much shorter than the electromagnetic (EM) wavelength are not "seen" by the radar. Even through the use of a filtered mss improves the accuracy of the GO2 model at nadir it remains very limited in incidence as corrections to the Gaussian shape of the scattering diagram must be quickly introduced. This can be partially compensated [12] by an incidence-dependent cutoff in the definition of the filtered mss but brings in an additional degree of arbitrariness. The main purpose of this paper is to propose an improved and robust version of GO2, termed GO4. The model now depends on the total instead of the filtered mss and the radar wavelength dependence is rendered through a diffraction term involving the curvature of the surface. As 
we will show, this makes it possible to achieve the accuracy of the Physical Optics (PO) with a very small number of parameters. This is useful in as much as it avoids the utilization of the sea surface spectrum which is bound to a specific model and can introduce further variability in the calculation of the backscattered power.

The GO4 model is by construction unpolarized and is therefore limited to the incidence angles where the polarization difference is negligible. It will certainly not outperform unified scattering models which have been proven to be accurate over a wide range of non-grazing incidence angles and, when combined with classical sea spectra, are in satisfactory overall agreement with experimental measurements (e.g. [5], [14], [15]). The main improvement brought by GO4 is to reduce the needed characterization of the unknown sea surface, including non-Gaussian effects, to the knowledge of the mere mss and msc parameters. As a result, the arbitrariness in the choice of a "preferred" spectral model is avoided, at least when estimating these parameters from experimental data. This gain in simplicity is obtained with quasi no loss in accuracy in the domain of validity of PO.

The introduction of a curvature correction to $\mathrm{GO} 2$ is not new but has followed in the past different approaches and results. The overall technique is based on perturbation expansion of the scattering amplitude or the electric or magnetic surface current ([9], [10], [11], [16], [17], [18]) with respect to a well-chosen small parameter combined with higher-order Taylor expansion or cumulant expansion of the structure function of wave elevations. Some of these results and their relation to our findings will be discussed in the core of the paper. More recently, an elegant mathematical approach was proposed based on hypothesizing a generalized Student form for the slope distribution [19]. We did not pursue in this way and chose a more physical approach even though the present results have been found consistent with this last approach.

The GO4 model is introduced in Section II for isotropic Gaussian surfaces and the evaluation of the curvature parameter is given in Section III. The generalization to anisotropic and non-Gaussian sea surfaces is provided in Section IV and V, respectively. Some comparisons with advanded analytical scattering 
models are given in Section VI and some applications of the GO4 model on various near-nadir data sets are discussed in Section VII.

\section{A SIMPLIFIED FORMALISM: THE GO4 MODEL}

In the microwave regime where sea surface roughness is large, the reference model for low-angle backscattering is the Physical Optics (PO) scalar approximation. It remains accurate as long as polarization effects remain negligible, that is in the first, say, 20-25 degrees of incidence away from nadir. We recall hereafter the geometry of the scattering problem. In the following we use the notation $a$ for the norm of any vector $\boldsymbol{a}$. We consider a rough interface $z=\eta(\boldsymbol{r})$ separating air (upper medium) from water (lower medium) and denote $\boldsymbol{r}=(x, y)$ the coordinate in the horizontal mean plane. The surface is illuminated from above by an incident monochromatic, linearly polarized, plane wave with wave vector $\boldsymbol{K}$ (corresponding to wavenumber $K$ ) at some incidence angle $\theta$ with respect to the vertical direction $\boldsymbol{z}$. In backscattering configuration it is convenient to introduce the Ewald vector $\boldsymbol{Q}=-2 \boldsymbol{K}$ together with its horizontal and vertical projections $\boldsymbol{Q}_{\boldsymbol{H}}$ and $Q_{z}$, respectively. Note that $Q_{H}=2 K \sin \theta$ and $Q_{z}=2 K \cos \theta$. The Normalized Radar Cross Section (NRCS) according to the PO approximation is expressed by the so-called Kirchhoff integral:

$$
\sigma_{P O}^{0}=K^{2} \sec ^{2}(\theta)|R|^{2} \frac{1}{\pi} \int d \boldsymbol{r} \mathrm{e}^{\mathrm{i} \boldsymbol{Q}_{\boldsymbol{H}} \cdot \boldsymbol{r}} \mathrm{e}^{-\frac{1}{2} Q_{z}^{2} S(\boldsymbol{r})} .
$$

Here $R$ is the Fresnel coefficient at normal incidence on the surface at rest and $S$ is the structure function of elevations:

$$
S(\boldsymbol{r})=2(\rho(\mathbf{0})-\rho(\boldsymbol{r})),
$$

which is trivially related to the roughness auto-correlation function $(\rho)$ or, what amounts to the same, to the wave number spectrum $(\Psi)$ through an inverse Fourier Transform: 


$$
\rho(\boldsymbol{r})=\int d \boldsymbol{k} \mathrm{e}^{\mathrm{i} \boldsymbol{k} \cdot \boldsymbol{r}} \Psi(\boldsymbol{k})
$$

For simplicity we assume in this section isotropicity of the wave number spectrum, so that the autocorrelation function and the PO NRCS are given by Bessel transforms:

$$
\rho(r)=\int_{0}^{\infty} d k 2 \pi k J_{0}(k r) \Psi(k)
$$

and

$$
\sigma_{P O}^{0}=K^{2} \sec ^{2}(\theta)|R|^{2} \int d r 2 r J_{0}\left(Q_{H} r\right) \mathrm{e}^{-\frac{1}{2} Q_{z}^{2} S(r)}
$$

For large Rayleigh parameter $\mathcal{R}=Q_{z}^{2} \rho(0)$, small lags have a dominant contribution to the integral and we may approximate the structure function by its asymptotic behavior about the origin:

$$
S(r) \simeq \frac{1}{2} \operatorname{mss} r^{2}
$$

where mss is the total mean square slope:

$$
\mathrm{mss}=\int_{0}^{\infty} d k 2 \pi k k^{2} \Psi(k)
$$

Insertion of this quadratic approximation of the structure function in the Kirchhoff integral leads to the classical GO2 approximation (e.g. [1]):

$$
\sigma_{G O 2}^{0}=\frac{|R|^{2}}{\mathrm{mss}} \sec ^{4}(\theta) \exp \left(-\frac{\tan ^{2} \theta}{\mathrm{mss}}\right)
$$

The GO2 model is parametrized solely by the mss parameter, which is well-defined and whose winddependence is well characterized. It is, however, in principle only valid in the limit of small wavelengths and becomes more and more accurate as the EM frequency is increased. At finite wavelength, a curvature correction is needed to incorporate the diffraction effects and the fact that the surface deviates from its 
tangent plane over a few EM wavelength. To this aim we push the Taylor expansion of the structure function at the next order using a fourth-order Taylor expansion of the Bessel function in (II.4):

$$
S(r) \simeq \frac{1}{2} \operatorname{mss} r^{2}-\frac{1}{32} \operatorname{msc} r^{4},
$$

where msc is the total mean square curvature:

$$
\operatorname{msc}=\int_{0}^{\infty} d k 2 \pi k k^{4} \Psi(k)
$$

In defining this last quantity we assume that the fourth moment of the spectrum is finite, which implies a high-frequency cut-off on the spectrum. Again, the approximation (II.9) is asymptotically valid in the limit of small lags and can be used to define the msc:

$$
\mathrm{msc}=\lim _{r \rightarrow 0} 32 \frac{\frac{1}{2} \mathrm{mss} r^{2}-S(r)}{r^{4}}
$$

At finite lag $r>0$ the total value of the msc is not reached but only a fraction of it, say $\beta(r)$ :

$$
\beta(r) \mathrm{msc}=32 \frac{\frac{1}{2} \mathrm{mss} r^{2}-S(r)}{r^{4}}
$$

Now the structure function in the Kirchhoff integral is only involved on a finite effective integration domain, say $\left[0, r_{e}\right]$ depending on the EM wavelength. On this given interval $\left[0, r_{e}\right]$, there is certainly a constant value $\mathrm{msc}_{e}=\beta$ msc which optimizes in some sense the quartic approximation of the structure function:

$$
S_{\beta}(r) \simeq \frac{1}{2} \operatorname{mss} r^{2}-\frac{1}{32} \operatorname{msc}_{e} r^{4}
$$

Note that this quartic approximation of the structure function at finite lag $r$ does not require the finiteness of the total msc, that is the existence of a finite limit in (II.11). We chose to optimize the value of $\beta$ in 
order to obtain the closest agreement with the PO NRCS at nadir whenever the structure function (II.2) is replaced by its quartic approximation (II.13). This amounts to equating:

$$
\int_{0}^{\infty}\left(\mathrm{e}^{-\frac{1}{2} Q_{z}^{2} S(r)}-\mathrm{e}^{-\frac{1}{2} Q_{z}^{2} S_{\beta}(r)}\right) r d r=0
$$

Now considering $S_{\beta}$ as a perturbation of $S$,

$$
S(r)=S_{\beta}(r)+\Delta S_{\beta}(r)
$$

with $Q_{z}^{2} \Delta S_{\beta}<<1$, we may rewrite:

$$
\int_{0}^{\infty} \mathrm{e}^{-\frac{1}{2} Q_{z}^{2} S(r)}\left(\mathrm{e}^{\frac{1}{2} Q_{z}^{2} \Delta S_{\beta}}-1\right) r d r=0
$$

To evaluate this integral we use the fact that the quartic term in the exponential should be small and can be linearized, that is:

$$
\mathrm{e}^{\frac{1}{2} Q_{z}^{2} \Delta S_{\beta}} \simeq \mathrm{e}^{\frac{1}{2} Q_{z}^{2}\left(S-\frac{1}{2} \mathrm{mss} r^{2}\right)}\left(1+Q_{z}^{2} \mathrm{msc}_{e} \frac{1}{64} r^{4}\right)
$$

This leads to the optimal msc:

$$
\mathrm{msc}_{e}=\frac{64}{Q_{z}^{2}} \frac{\int_{0}^{\infty} \mathrm{e}^{-\frac{1}{2} Q_{z}^{2} S(r)}\left(\mathrm{e}^{\frac{1}{2} Q_{z}^{2}\left(S-\frac{1}{2} m s s r^{2}\right)}-1\right) r d r}{\int_{0}^{\infty} \mathrm{e}^{-\frac{1}{4} Q_{z}^{2} \mathrm{mss} r^{2}} r^{5} d r}
$$

where the structure function is evaluated from (II.2) and (II.3) and $Q_{z}=2 K$ is taken at nadir. To evaluate the Kirchhoff integral with the modified structure function (II.13), we again take advantage of the small magnitude of the quartic term in the exponential which can thus be linearized. This leads to the following approximation for the PO integral:

$$
\begin{aligned}
\sigma^{0} & \simeq K^{2} \sec ^{2}(\theta)|R|^{2} \frac{1}{\pi} \int d \boldsymbol{r} \mathrm{e}^{\mathrm{i} \boldsymbol{Q}_{\boldsymbol{H}} \cdot \boldsymbol{r}} \mathrm{e}^{-\mathrm{mss} Q_{z}^{2} \frac{r^{2}}{4}} \\
& \times\left(1+\frac{1}{64} \operatorname{msc} r^{4}\right)
\end{aligned}
$$


The evaluation of this integral can be performed routinely and leads to the following correction to the $\mathrm{GO} 2$ formula:

$$
\begin{aligned}
& \sigma_{G O 4}^{0}=\sigma_{G O 2}^{0} \times \\
& {\left[1+\frac{\mathrm{msc}_{e}}{16 K^{2} \mathrm{mss}^{2} \cos ^{2} \theta}\left(\frac{\tan ^{4} \theta}{\mathrm{mss}^{2}}-4 \frac{\tan ^{2} \theta}{\mathrm{mss}}+2\right)\right],}
\end{aligned}
$$

where $\sigma_{G O 2}^{0}$ is the GO2 NRCS with total mss.

We refer to this approximation as the "GO4" approximation as opposed to the GO2 model which involves only a quadratic approximation of the structure function. We call the modified curvature $\mathrm{msc}_{e}=$ $\beta$ msc the effective mean square curvature of the surface, which depends on the EM wavelength. This formula and the GO4 terminology were already introduced in [20] but at that time only the total and not the effective msc was considered. Note that formula (II.20) with the total curvature is equivalent to the diffraction correction developed in [18] at nadir $(X=0)$ using the iterated magnetic current integral equation.

\section{THE EFFECTIVE MEAN SQUARE CURVATURE}

The GO4 approximation relies on two parameters only, namely the total mean square slope mss and the effective mean square curvature $\mathrm{msc}_{e}$. The total mss is a meaningful quantity because it quantifies the exchange surface between ocean and atmosphere or, in mathematical terms, because the decrease of the sea spectrum ensures the convergence of the second spectral moment. On the contrary, the notion of total curvature is ill-defined because it refers to the "sharpness" of wave which is ever increasing at small scales. In mathematical terms, the total msc is the fourth moment of the surface spectrum (which typically falls-off in $k^{-4}$ ) and is thus dramatically sensitive to the chosen high-frequency cut-off. This raises the issue of "where the spectrum should stop" and questions the microscopic nature of the surface. Hence, it is only the curvature at a finite scale which is meaningful. However, as it is well known, the EM scattering process performs a natural filter at the scales which cannot be "seen" by the probing EM wavelength. It is therefore clear than the total msc is not the relevant quantity to consider for the scattering process. It is 
more relevant to consider the effective msc of the rough surface filtered at the given EM frequency, even though the EM filtering process is somewhat more complex than a sharp cut-off on spatial frequencies. For these reasons, the radar wavelength dependence which is often used to improve the GO2 model has been transferred from the slope to the curvature parameter in the GO4 model. Note that the effective msc does not depend on the incidence angle as it is evaluated by matching the NRCS at nadir. The effective msc should be of the order of magnitude of the fourth moment of the surface spectrum truncated at the EM wavenumber. We therefore define the dimensionless parameter $\alpha$ by:

$$
\operatorname{msc}_{e}=\int_{0}^{\alpha K} 2 \pi k k^{4} \Psi(k) d k,
$$

which we expect to be close to unity. This parameter has the advantage over the alternative parameter $\beta$ that it does not require the knowledge of the full msc. Note also that $\alpha$, like $\operatorname{msc}_{e}$, is independent on the incidence angle.

We have calculated the parameters $\alpha$ and $\mathrm{msc}_{e}$ (from II.18) for three different omnidirectional wave number spectra, referred to by the name of their first author: Elfouhaily unified spectrum [21], Bringer remote sensing spectrum [22] and Kudryavtsev physical spectrum [23], [24]. Recently, some refined shortwave spectral models have been proposed, such as a roughness spectrum based on field measurements including the effect of swell [25] or an improved directional spectrum based on stereo-photography [26]. However, in the present study we will limit ourselves to the simple aforementioned omnidirectional spectra.

Figures 1 and 2 show the evolution of the parameters $\alpha$ and $\operatorname{msc}_{e}$ with the EM wavelength and wind speed. The effective msc has been evaluted from (II.18) at nadir and $\alpha$ from (III.21). Numerical convergence tests have been performed on the space and frequency sampling rate to produce an accurate value of these parameters.

As expected, the effective msc grows importantly with both EM frequency and wind speed and ranges over a few decades. Important relative variations (up to 20-30\%) are observed between different spectral models. The cut-off parameter $\alpha$, on the contrary, remains quite stable and increases only slightly with 
wind speed and inverse frequency. In average over different incidence angles and spectral models we have $\alpha \simeq 1$ in Ka band, $\alpha \simeq 1.5$ in $\mathrm{Ku}$ band and $\alpha \simeq 2.25$ in $\mathrm{C}$ band.

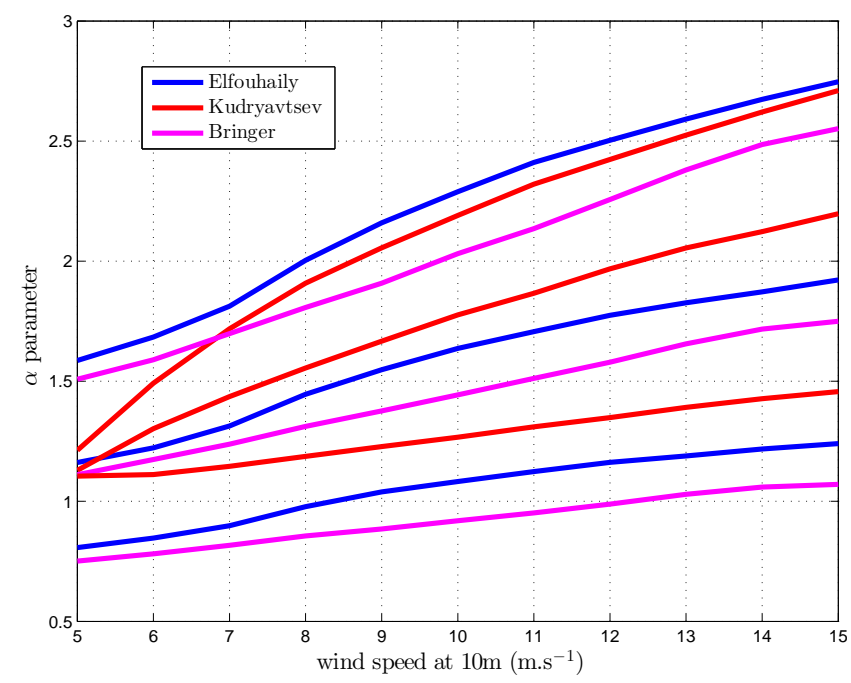

Fig. 1: Evolution of the cutoff parameter $\alpha$ with wind speed and EM frequency for three different models of omnidirectional spectrum: Elfouhaily (blue), Kudryavtsev (red) and Bringer (magenta). Three frequencies are shown, namely $\mathrm{C}$ band (upper curves), Ku band (middle curves) and Ka band (lower curves). In Ka band, the value of $\alpha$ is close to 1, meaning that the effective msc is approximately the total msc truncated at the EM wavelength.

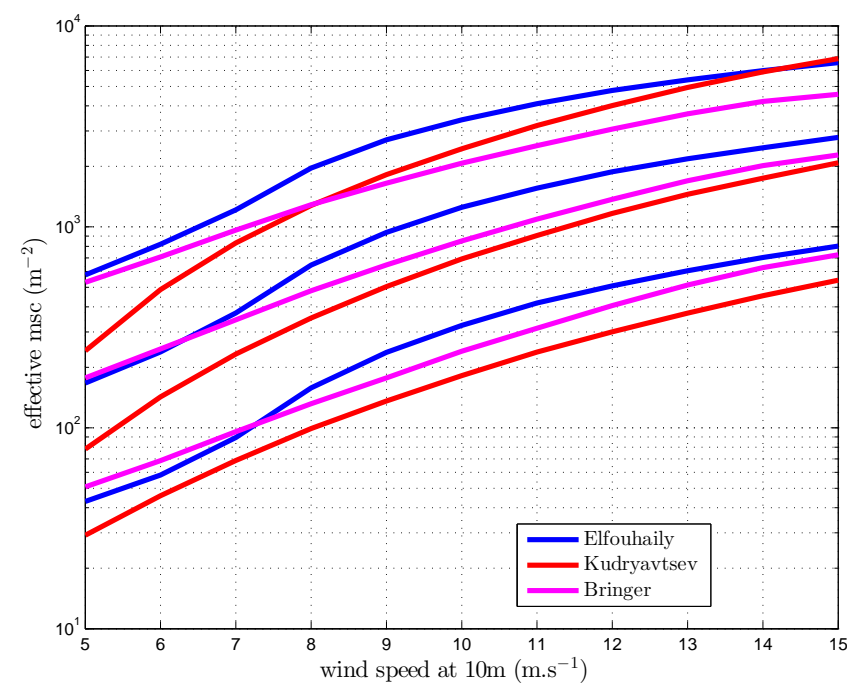

Fig. 2: Same as Figure 1 for the effective msc except that $\mathrm{C}$ band is lower curve and Ka band is upper curve.

Figures 3,4 and 5 show the isotropic NRCS according to GO4 with the predicted value of the cut-off parameter in the different bands: $\alpha=1.25$ in Ka band, $\alpha=1.89$ in $\mathrm{Ku}$ band and $\alpha=2.64$ in $\mathrm{C}$ band. The calculation has been performed with an omnidirectional Elfouhaily spectrum at wind speed $=10 \mathrm{~m} / \mathrm{s}$. A comparison with $\mathrm{GO} 2$ and $\mathrm{PO}$ is given. The $\mathrm{GO} 2$ with filtered mss is also given for reference, in which 
the classical K/3 cut-off ([27]) is employed. Even though it brings a significant improvement over the GO2 with full mss, it is still about $1 \mathrm{~dB}$ away from $\mathrm{PO}$ at nadir. At moderate wind speed $(10 \mathrm{~m} / \mathrm{s})$, an excellent agreement is found between PO and GO4 in the first 15 degrees in Ka band, the first 12 degrees in $\mathrm{Ku}$ band and the first 10 degrees in $\mathrm{C}$ band.

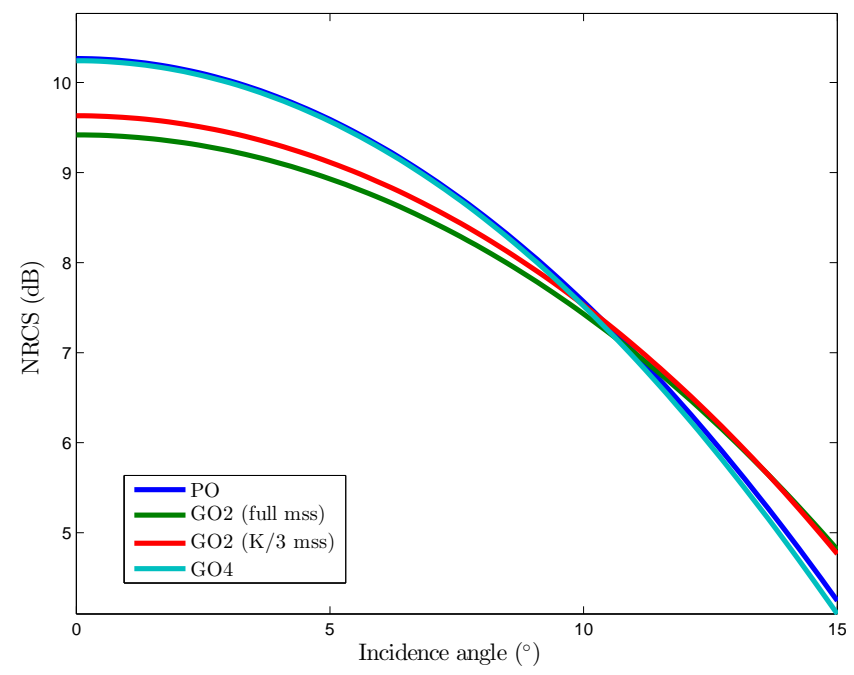

Fig. 3: Comparison of PO, GO2 and GO4 in Ka band with $\alpha=1.26$ for an omnidirectional Elfouhaily spectrum with wind speed $=10 \mathrm{~m} / \mathrm{s}$

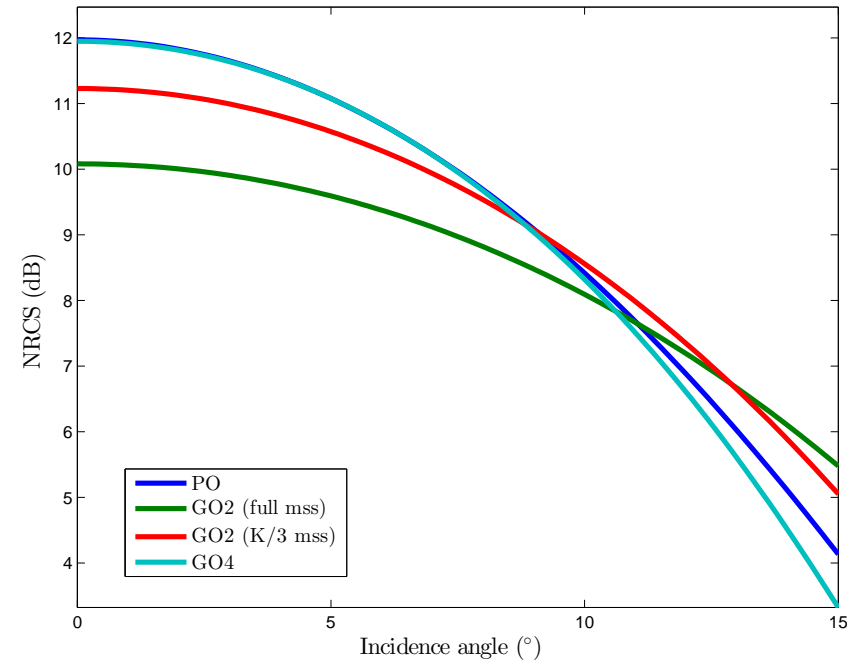

Fig. 4: Comparison of PO, GO2 and GO4 in Ku band with $\alpha=1.89$ for an omnidirectional Elfouhaily spectrum with wind speed $=10 \mathrm{~m} / \mathrm{s}$ 


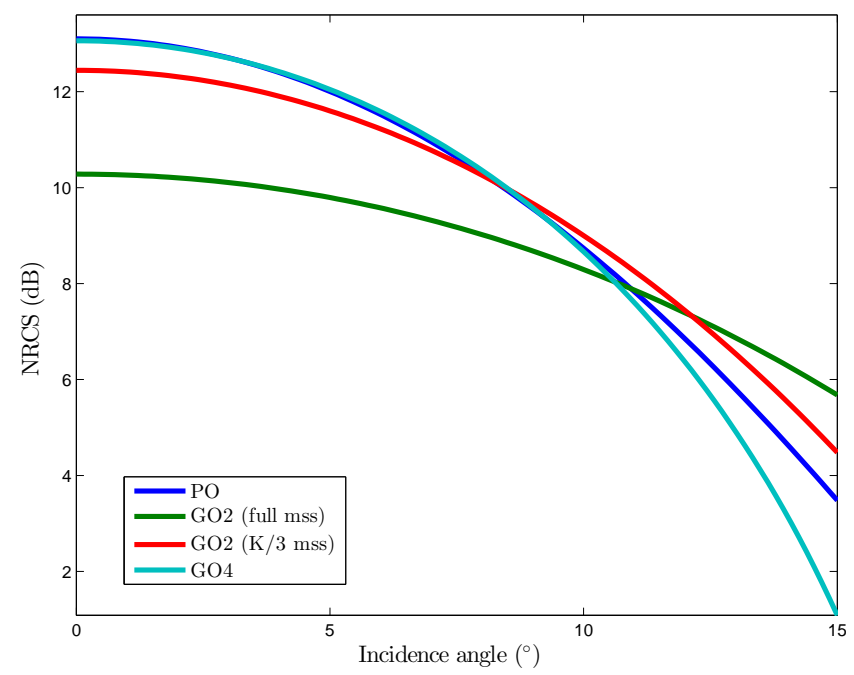

Fig. 5: Comparison of PO, GO2 and GO4 in $\mathrm{C}$ band with $\alpha=2.64$ for an omnidirectional Elfouhaily spectrum with wind speed $=10 \mathrm{~m} / \mathrm{s}$

Note that the agreement between GO4 and PO improves as the radar frequency is increased. This is consistent with the high-frequency approximation used in defining GO4, in which only the asymptotic behavior (II.9) of the structure function at small lags is considered. More generally, the condition of validity of the GO4 is the same as the Physical Optics, namely a large value of the Rayleigh parameter $\mathcal{R}$. Hence, high wind speeds are more favorable to the GO4 model, at a given radar frequency.

\section{Anisotropic CASE}

We now develop the GO4 model in the general framework of anisotropic surfaces. Similar calculations have already been derived by one of the authors [28] in the high-frequency limit of the PO but the distinction between effective and total msc was not considered. We recall the expression of the directional GO2, where $\theta$ is the incidence angle with respect to the vertical axis and $\varphi$ is the azimuth angle with respect to the $\mathrm{x}$-axis:

$$
\begin{aligned}
\sigma_{G O 2}^{0}(\theta, \varphi) & =\frac{|R|^{2}}{\sqrt{\mathrm{mss}_{x} \mathrm{mss}_{y}}} \sec ^{4}(\theta) \\
& \times \exp \left(-\frac{\tan ^{2} \theta}{2}\left(\frac{\cos ^{2} \varphi}{\operatorname{mss}_{x}}+\frac{\sin ^{2} \varphi}{\operatorname{mss}_{y}}\right)\right),
\end{aligned}
$$

with $\operatorname{mss}_{x}, \operatorname{mss}_{y}$ the directional slopes: 


$$
\operatorname{mss}_{x}=\int k_{x}^{2} \Psi(\boldsymbol{k}) d \boldsymbol{k}, \operatorname{mss}_{y}=\int k_{y}^{2} \Psi(\boldsymbol{k}) d \boldsymbol{k}
$$

The fourth-order Taylor expansion of the structure function is easily found to be:

$$
\begin{aligned}
S(x, y) & =\operatorname{mss}_{x} x^{2}+\operatorname{mss}_{y} y^{2} \\
& -\frac{1}{12}\left(\operatorname{msc}_{x} x^{4}+6 \operatorname{msc}_{x y} x^{2} y^{2}+\operatorname{msc}_{y} y^{4}\right),
\end{aligned}
$$

where $\mathrm{msc}_{x}, \mathrm{msc}_{y}, \mathrm{msc}_{x y}$ are the directional curvatures:

$$
\begin{aligned}
\operatorname{msc}_{x} & =\int k_{x}^{4} \Psi(\boldsymbol{k}) d \boldsymbol{k}, \\
\operatorname{msc}_{y} & =\int k_{y}^{4} \Psi(\boldsymbol{k}) d \boldsymbol{k}, \\
\operatorname{msc}_{x y} & =\int k_{x}^{2} k_{y}^{2} \Psi(\boldsymbol{k}) d \boldsymbol{k}
\end{aligned}
$$

Note that the total mss and msc are given by:

$$
\begin{aligned}
& \mathrm{mss}=\int k^{2} \Psi(\boldsymbol{k}) d \boldsymbol{k}=\mathrm{mss}_{x}+\mathrm{mss}_{y} \\
& \mathrm{msc}=\int k^{4} \Psi(\boldsymbol{k}) d \boldsymbol{k}=\mathrm{msc}_{x}+\mathrm{msc}_{y}+2 \mathrm{msc}_{x y}
\end{aligned}
$$

Straightforward calculations very similar to those employed in the isotropic case lead to the following formula for the directional GO4 approximation:

$$
\begin{aligned}
& \sigma_{G O 4}^{0}(\theta, \varphi)=\sigma_{G O 2}^{0}(\theta, \varphi)\left\{1+\frac{1}{96 K^{2} \cos ^{2} \theta}\right. \\
& \times\left[\frac{6 \mathrm{msc}_{x y}}{\operatorname{mss}_{x} \operatorname{mss}_{y}} H_{2}(X) H_{2}(Y)\right. \\
& \left.\left.+\frac{\operatorname{msc}_{x}}{\operatorname{mss}_{x}^{2}} H_{4}(X)+\frac{\operatorname{msc}_{y}}{\operatorname{mss}_{y}^{2}} H_{4}(Y)\right]\right\}
\end{aligned}
$$

where the variables $X, Y$ are given by:

$$
X=\frac{\tan \theta \cos \varphi}{\sqrt{\mathrm{mss}_{x}}}, Y=\frac{\tan \theta \sin \varphi}{\sqrt{\mathrm{mss}_{y}}}
$$


and $H_{n}$ are the Hermite polynomials:

$$
H_{n}(u)=(-1)^{n} e^{u^{2} / 2} \frac{d^{n}}{d u^{n}} e^{-u^{2} / 2}
$$

It is interesting to consider the directional GO4 formula (IV.27) in the particular case of a bi-harmonic spectrum, such as Elfouhaily unified spectrum:

$$
\Psi(k, \varphi)=\frac{1}{2 \pi k} \Psi_{0}(k)(1+\Delta(k) \cos (2 \varphi))
$$

In addition to the total or isotropic mss and msc ( referred to by a " $\mathrm{i}$ " subscript in the following equations), it is useful to introduce the anisotropic mss and msc, referred to by a "a" subscript:

$$
\begin{aligned}
\operatorname{mss}_{i} & =\int k^{2} \Psi_{0}(k) d k ; \operatorname{mss}_{a}=\int k^{2} \Psi_{0}(k) \Delta(k) d k \\
\operatorname{msc}_{i} & =\int k^{4} \Psi_{0}(k) d k ; \operatorname{msc}_{a}=\int k^{4} \Psi_{0}(k) \Delta(k) d k
\end{aligned}
$$

We then have the simple relations:

$$
\begin{aligned}
\mathrm{mss}_{x / y} & =\frac{1}{2}\left(\operatorname{mss}_{i} \pm \frac{\mathrm{mss}_{a}}{2}\right) \\
\mathrm{msc}_{x / y} & =\frac{1}{4}\left(\frac{3}{2} \mathrm{msc}_{i} \pm \mathrm{msc}_{a}\right) \\
6 \mathrm{msc}_{x y} & =\mathrm{msc}_{x}+\mathrm{msc}_{y}
\end{aligned}
$$

This reduces the number of slopes and curvature parameters from 5 to 4 and allows it to calculate them through one-dimensional integrals only.

\section{NON-GAUSSIAN CORRECTIONS}

In the case of a weakly non-Gaussian surface, the Kirchhoff integral admits corrective terms corresponding to the cumulant expansion of the two-point characteristic function: 


$$
\begin{aligned}
& \sigma_{P O}^{0}=K^{2} \sec ^{2}(\theta)|R|^{2} \\
& \times \frac{1}{\pi} \int d \boldsymbol{r} \mathrm{e}^{\mathrm{i} \boldsymbol{Q}_{\boldsymbol{H}} \cdot \boldsymbol{r}} \mathrm{e}^{-\frac{1}{2} Q_{z}^{2} S(\boldsymbol{r})+\mathrm{i} \frac{1}{6} \mathrm{Q}_{\mathrm{z}}^{3} \mathrm{~S}_{3}(\boldsymbol{r})+\frac{1}{24} \mathrm{Q}_{\mathrm{z}}^{4} \mathrm{~S}_{4}(\boldsymbol{r})},
\end{aligned}
$$

where $S_{3}$ and $S_{4}$ are the skewness and kurtosis function, respectively:

$$
\begin{aligned}
& S_{3}(\boldsymbol{r})=\left\langle(\eta(\boldsymbol{r})-\eta(0))^{3}\right\rangle \\
& S_{4}(\boldsymbol{r})=\left\langle(\eta(\boldsymbol{r})-\eta(0))^{4}\right\rangle-3\left(\left\langle(\eta(\boldsymbol{r})-\eta(0))^{2}\right\rangle\right)^{2}
\end{aligned}
$$

The skewness and kurtosis functions are governed by the skewness and excess kurtosis of slopes for small arguments:

$$
\begin{aligned}
S_{3}(x, y) & =\lambda_{30} \operatorname{mss}_{x}^{3 / 2} x^{3}+\lambda_{03} \operatorname{mss}_{y}^{3 / 2} y^{3} \\
& +3 \lambda_{21} \operatorname{mss}_{x} \operatorname{mss}_{y}^{1 / 2} x^{2} y+3 \lambda_{12} \operatorname{mss}_{y} \operatorname{mss}_{x}^{1 / 2} x y^{2} \\
S_{4}(x, y) & =\lambda_{40} \operatorname{mss}_{x}^{2} x^{4}+\lambda_{04} \operatorname{mss}_{y}^{2} y^{4} \\
& +6 \lambda_{22} \operatorname{mss}_{x} \operatorname{mss}_{y} x^{2} y^{2}
\end{aligned}
$$

where the dimensionless coefficients $\lambda_{m n}$ are defined by:

$$
\lambda_{m n}=\frac{\left\langle\left(\partial_{x} \eta\right)^{m}\left(\partial_{y} \eta\right)^{n}\right\rangle}{\left\langle\left(\partial_{x} \eta\right)^{2}\right\rangle^{m / 2}\left\langle\left(\partial_{y} \eta\right)^{2}\right\rangle^{n / 2}}
$$

We do not detail the calculations leading to the GO4 NRCS in the non-Gaussian case, as they are very similar to those employed in the Gaussian case. The Taylor expansions of $S_{3}$ and $S_{4}$ can be combined with the fourth-order expansion (IV.24) of the structure function and injected in the non-Gaussian expression (V.33) of the PO NRCS. The terms of order 3 and 4 in the exponential are assumed small and linearized out of the exponential. This reduces the Kirchhoff integral to a two-dimensional Fourier Transform of a Gaussian function multiplied by a bi-variate polynomial of fourth degree. In this way we obtain the GO4 formula with skewness and kurtosis correction: 


$$
\begin{gathered}
\sigma_{G O 4}^{0}=\sigma_{G O 2}^{0} \times \\
\left\{1+\frac{1}{24 Q_{z}^{2}}\left[6\left(\frac{\mathrm{msc}_{x y}}{\operatorname{mss}_{x} \mathrm{mss}_{y}}+\lambda_{22} Q_{z}^{2}\right) H_{2}(X) H_{2}(Y)\right.\right. \\
\left.+\left(\frac{\mathrm{msc}_{x}}{\operatorname{mss}_{x}^{2}}+\lambda_{40} Q_{z}^{2}\right) H_{4}(X)+\left(\frac{\operatorname{msc}_{y}}{\operatorname{mss}_{y}^{2}}+\lambda_{04} Q_{z}^{2}\right) H_{4}(Y)\right] \\
+\frac{1}{6}\left[3 \lambda_{21} H_{2}(X) H_{1}(Y)+3 \lambda_{12} H_{1}(X) H_{2}(Y)\right. \\
\left.\left.+\lambda_{30} H_{3}(X)+\lambda_{03} H_{3}(Y)\right]\right\}
\end{gathered}
$$

A resembling formula was proposed in [16] based on a cumulant expansion of the structure function. This last result is, however, different in as much as fourth-order terms in the polynomial expansion stem uniquely from non-vanishing cumulants (that is the very non-Gaussian nature) of the slope distribution and not from the proper geometrical curvature of the surface. An interesting particular case is the isotropic surface for which we have:

$$
\begin{aligned}
\operatorname{mss}_{x} & =\operatorname{mss}_{y}=\frac{1}{2} \mathrm{mss} \\
\mathrm{msc}_{x} & =\mathrm{msc}_{y}=3 \mathrm{msc}_{x y}=\frac{3}{8} \mathrm{msc}_{e} \\
\lambda_{30} & =\lambda_{03}=\lambda_{12}=\lambda_{21}=0 \\
\lambda_{40} & =\lambda_{04}=3 \lambda_{22}=\lambda_{4}
\end{aligned}
$$

After some calculations we find that the expression (V.37) can be simplified to:

$$
\begin{gathered}
\sigma_{G O 4}^{0}=\sigma_{G O 2}^{0}\left[1+\left(\frac{\mathrm{msc}_{e}}{4 Q_{z}^{2} \mathrm{mss}^{2}}+\frac{\lambda_{4}}{6}\right)\right. \\
\left.\left(\frac{\tan ^{4} \theta}{\mathrm{mss}^{2}}-4 \frac{\tan ^{2} \theta}{\mathrm{mss}}+2\right)\right]
\end{gathered}
$$

Hence we recover the isotropic NRCS of the Gaussian case by augmenting the effective msc with the contribution of the excess kurtosis, that is with obvious notations:

$$
\left.\operatorname{msc}_{e}\right|_{N G}=\left.\operatorname{msc}_{e}\right|_{G}+\frac{2}{3} \lambda_{4} \mathrm{mss}^{2} Q_{z}^{2}
$$


Note that the non-Gaussian effective msc is slightly dependent on the incidence angle through the corrective term. Figure 6 shows the respective contributions of the Gaussian msce and its non-Gaussian correction with the value $\lambda_{4}=0.4$ corresponding to the quasi-constant value found by Cox and Munk [29] in their famous experiment (we discard directionality with respect to wind vector). The kurtosis correction becomes important in Ka band and can increase the effective msc by about $50 \%$.

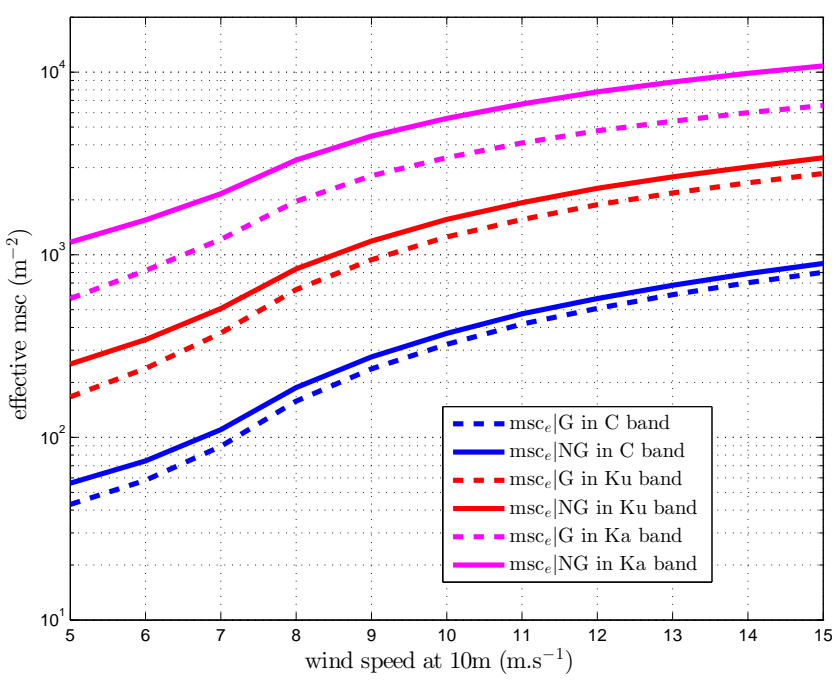

Fig. 6: Gaussian (G) and Non-Gaussian (NG) msc for the omnidirectional Elfouhaily spectrum in different bands.

In the introduction of the kurtosis correction to the effective msc, it is important to keep in mind that the value 0.4 experimentally reported by Cox and Munk is not only due to the peakedness of the slope distribution (very small and very large slopes being more frequent than predicted by the Gaussian distribution) but also on the compound nature of sea slope statistics as was explained in [30]. Hence, fluctuations of statistics across the different sea patches results in an augmentation of the effective msc based on a statistically homogeneous model.

\section{COMPARISON WITH OTHER APPROXIMATE MODELS}

\section{A. Advanced analytical models}

There has been a certain number of unified scattering models proposed in the last two decades (see e.g. [2] for a review). We will here briefly discuss some of them in connection to the GO4 model. First note that the GO4 model is a simplification of the Physical Optics model and, as such, is not sensitive 
to polarization. Hence the GO4 model cannot perform better that advanced analytical models which have a polarization dependence, such as e.g. the Small-Slope Approximation (SSA, [3]), the Local Curvature Approximation (LCA, [31]), the Weighted Curvature Approximation (WCA, [8]), the Resonant Curvature Approximation (RCA, [6]) or the cut-off invariant Two-Scale Model (GO-SSA, [7]), to cite only a few. In the upper microwave bands starting from the $\mathrm{C}$ band, the sea surface has very large roughness and the Physical Optics is the reference model at low angles. Hence, we expect the GO4 model to be accurate in the nadiral region where polarization effects can be neglected, the range of incidence over which this is verified depending on sea state and frequency band (that is on the Rayleigh parameter $\mathcal{R}$ ). The aforementioned advanced approximations are unified scattering models, that is in principle capable of handling all types of geometry and sea states. We will not describe them in details and refer to the cited publications for their description. The main general underlying principle of such models is their compliancy with the fundamental asymptotic models in the appropriate limits, that is consistency with the Small Pertubation Expansion for small roughness and with the Physical Optics for large Rayleigh parameter. This automatic adjustment to the relevant asymptotic model is reached through the introduction of either an extra nonlocal frequency kernel in the Kirchhoff integral (SSA, LCA, RCA), a local slope-dependent kernel (WCA) or facet tilting (GO-SSA). These models have proven to be useful in the comparison with experimental data combining various angles of incidences and radar frequency (e.g. [14], [22], [15]). As these models are essentially based on heuristic grounds and devised to be generalist, they might be slightly less accurate (by a fraction of $\mathrm{dB}$ ) at the very nadir than a mere PO model. Hence, when it comes to evaluating data in a restricted range of incidence about nadir it both simpler and safer to use a simple GO4 model.

Figure 7 shows the comparison of the different models in different bands for an isotropic Elfouhaily spectrum. The different models stay within at most $0.5 \mathrm{~dB}$ from each other in the range of incidence where GO4 was found to reproduce accurately PO (at $10 \mathrm{~m} / \mathrm{s}$ wind speed, this corresponds to the first 10 degrees in $\mathrm{C}$ band, the first 12 degrees in $\mathrm{Ku}$ band and the first 15 degrees in Ka band). 


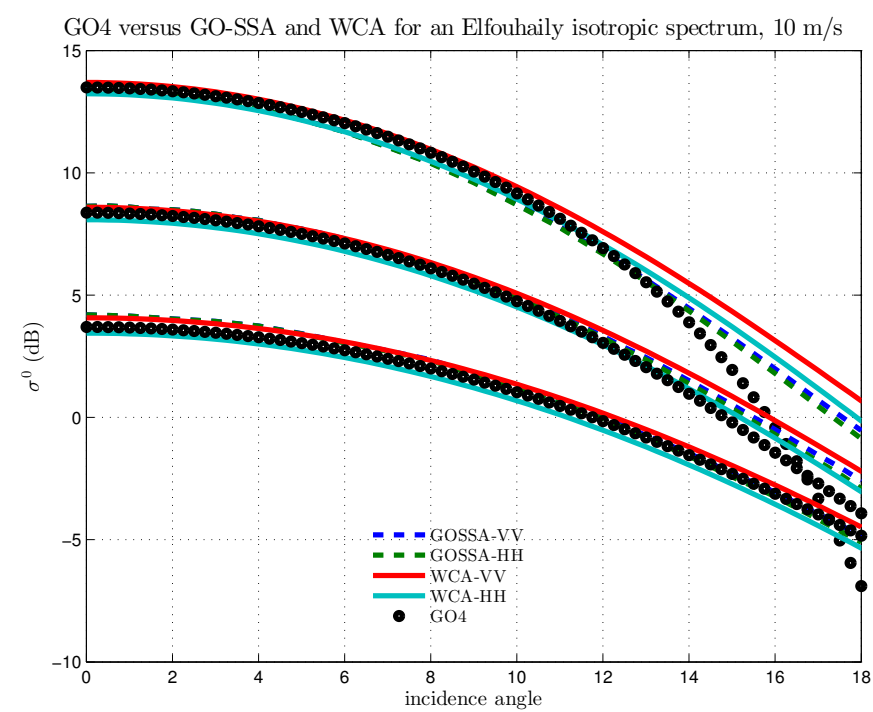

Fig. 7: Comparison of the GO4 model to GO-SSA and WCA models for an isotropic Elfouhaily spectrum by $10 \mathrm{~m} / \mathrm{s}$ wind speed in C (upper curves), $\mathrm{Ku}$ (middle curves) and Ka (lower curves) bands. For better visibility the Ku and Ka NRCS have been offset by -3 $\mathrm{dB}$ and $-6 \mathrm{~dB}$, respectively.

\section{B. Non-Gaussian Physical Optics}

It is well known (e.g. [20]) that non-Gaussian corrections of the surface statistics are important in interpreting the near-nadir NRCS. Non-Gaussian corrections enter in the PO model through the addition of higher-order structure functions of the field of elevations. The dominant contribution for the omnidirectional NRCS is the so-called peakedness correction involving the fourth-order structure function, which is itself related to the excess kurtosis of surface slope, $\lambda_{4}$. We refer to [20] for a detailed derivation of the non-Gaussian PO model. Figure 8 shows the comparison between the non-Gaussian PO (PO-NG) and the non-Gaussian (GO4-NG) in different bands for an isotropic Elfouhaily spectrum at $10 \mathrm{~m} / \mathrm{s}$ and the same value $\lambda_{4} \simeq 0.4$. A conclusion similar to the previous case holds, namely that the different models agree as long as the Gaussian GO4 model is able to reproduce accurately the Gaussian PO. 


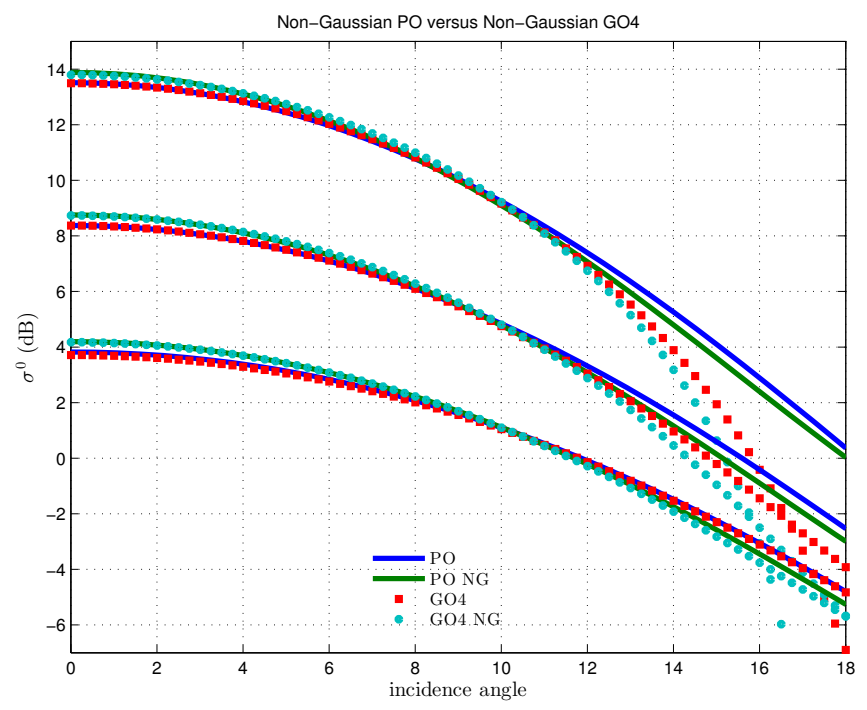

Fig. 8: Comparison of the non-Gaussian GO4 model with the non-Gaussian Physical Optics model of [20] for an isotropic Elfouhaily spectrum by $10 \mathrm{~m} / \mathrm{s}$ wind speed in $\mathrm{C}$ (upper curves), Ku (middle curves) and Ka (lower curves) bands. For better visibility the Ku and Ka NRCS have been offset by $-3 \mathrm{~dB}$ and $-6 \mathrm{~dB}$, respectively.

\section{ESTIMATION OF THE MSS AND MSC FROM EXPERIMENTAL DATA}

\section{A. Estimation procedure}

The main advantage of the two-parameter GO4 model is the possibility to estimate directly the total mss, which is an intrinsic parameter of the sea surface, and not the "mss seen by the radar" which depends on the EM wavelength. In addition, it provides an accurate parametrization of the backscattering cross-section in a wide angular domain around nadir with the additional knowledge of the effective msc.

For this illustration of the GO4 concept, we will restrict the consideration to the omnidirectional NRCS, a more detailed study being left for further work. We recall that this quantity is obtained by averaging all possible azimuthal direction $\varphi$ at the same incidence angle $\theta$ :

$$
\sigma_{o m n i}^{0}(\theta)=\frac{1}{2 \pi} \int_{0}^{2 \pi} d \varphi \sigma^{0}(\theta, \varphi)
$$

The omnidirectional NRCS will be treated with the isotropic GO4 model, even though this introduce small bias in the estimation of the mss and msc (see the discussion further). 
Our analysis will be based on several near-nadir data sets from the literature. Since the different data sets are well-documented we will not enter in their description and simply refer to the main publications. We will use the Ka-band airborne scanning radar altimeter (SRA) data of the Southern Ocean Waves Experiment (SOWEX, [32], [33]), the $\mathrm{Ku} / \mathrm{C}$ Jason2 altimeter data, data from the $\mathrm{Ku}$ band precipitation radar from the Tropical Rainfall Measuring Mission [34], [35] and the Ka-band airborne scatterometer data described in [36].

The difficulty in evaluating the mss and msc parameters from spaceborne or airborne data is the uncertainty linked to the data calibration. However, whenever a certain range of incidence angle is available, the joint estimation of mss and msc can be performed on the basis of relative values (i.e. non calibrated) of the NRCS. In that case, the parameter estimation is obtained upon minimization of the following cost function in a certain range of incidence:

$$
\Phi=\sum_{\theta<\theta_{\max }}\left|\sigma_{\text {data }}^{0}(\theta)-\sigma_{G O 4}^{0}(\mathrm{mss}, \mathrm{msc}, \theta)\right|^{2},
$$

where the NRCS are taken in $\mathrm{dB}$ and normalized by their value at nadir. This has been done for the SRA data in Ka band and the TRMM data in Ku band for which ranges of incidence of $0-25$ degrees and $0-18$ degrees, respectively, are available. It is important to note that the accuracy of the parameter estimation is slightly dependent on the chosen range of incidence. It should be chosen as large as possible in order to better separate the quartic behavior (GO4) from the quadratic behavior (GO2) with respect to the variable $\tan \theta$ but, on the other hand, should respect the validity domain of the GO4 approximation. This sensibility of the estimated shape parameters to the incidence span has been known for a long time in the case of the estimation of the single radar-mss from a GO2 model (this is discussed in detail in [37]). It requires some a priori knowledge of the incidence span over which the model is expected to hold. From the systematic analysis of section III with synthetic data at various wind speeds (exemplified on Figures 3, 4 and 5) we have seen that this validity domain increases with both EM frequency and wind speed. 
For altimeter data where only the nadir NRCS is available we will rely on its absolute level. The effective msc is then evaluated from Cox and Munk mss [29], which we abbreviate to "CM-mss" . This has been done with Jason2 data in $\mathrm{C}$ and $\mathrm{Ku}$ band as well as the $\mathrm{Ka}$ band airborne measurements from [36].

The values of msc inverted from experimental data have been systematically compared with those calculated from the spectral models presented in section III, except for the Bringer spectral model. This last model cannot provide a fair evaluation as it has been constructed using partly the same data sets [22].

\section{B. TRMM}

Figure 9 shows the total mss inverted from the omnidirectional TRMM NRCS using either the simple GO2 model or the joint inversion of mss and msc with the GO4 model. The first 10 degrees of the diagram have been used for the fit as it is the assumed minimal domain of validity of the GO4 model in $\mathrm{Ku}$ band. Error bars around the GO4 mss and msc shows the effect of changing by \pm 1 degree the interval of estimation. A comparison is given with the total CM-mss and the radar-filtered mss calculated with Elfouhaily spectral model using the usual frequency cut-off at $K / 3$. As seen, the mss obtained from the mere GO2 model is close to the radar-filtered mss while the mss estimated from GO4 is in excellent agreement with the total mss. The effective msc estimated with GO4 is shown on Figure 10 and is found in excellent agreement with the effective msc derived from the analytical spectra. In the calculation of the effective msc we have used formula (II.18) together with the non-Gaussian correction (V.40). 


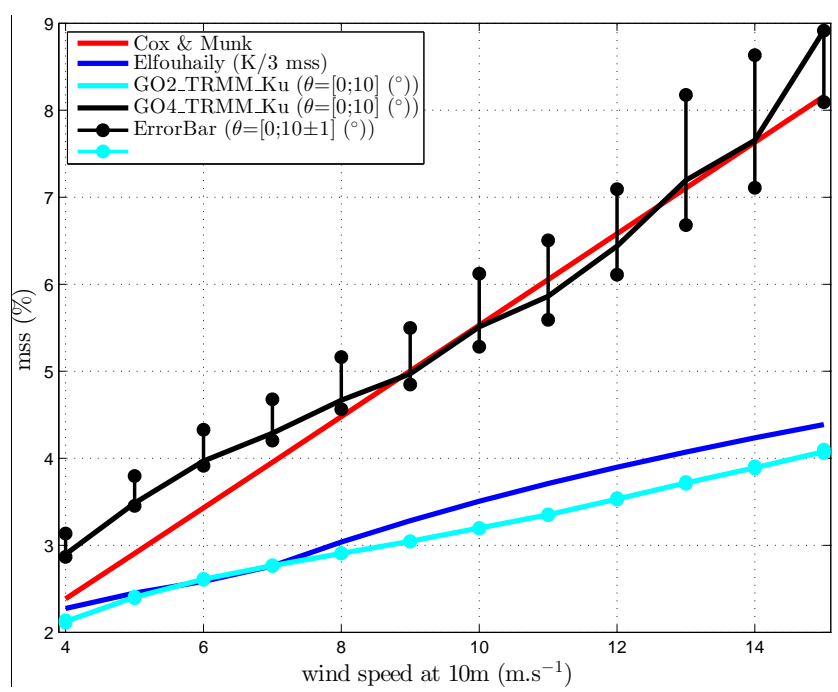

Fig. 9: Estimation of the mss with the GO2 and GO4 model for the TRMM Ku data. A comparison is given with the filtered mss for different spectra. The error bars indicate the sensibility to a small change in the chosen angular domain for the GO2 and GO4 minimization process (VII.42).

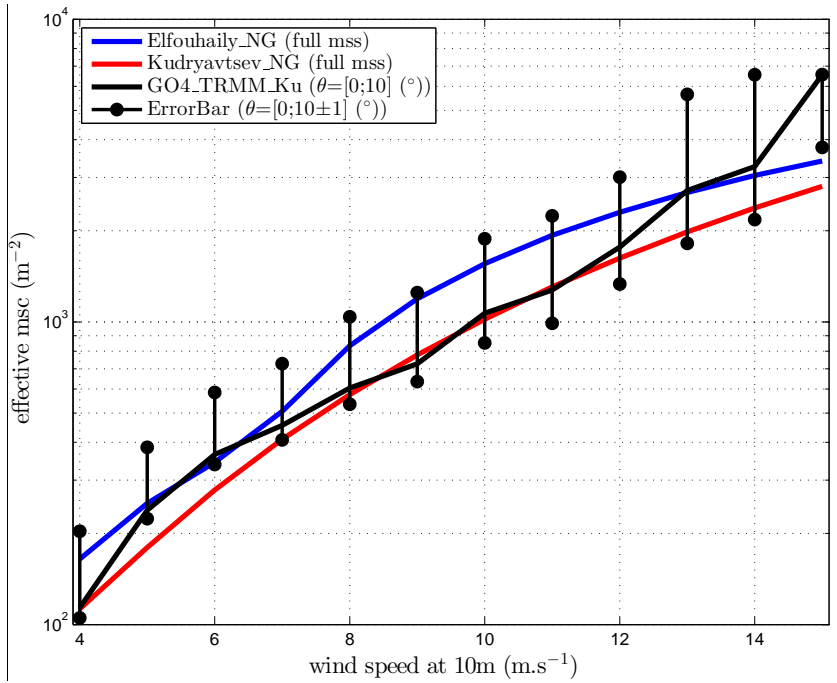

Fig. 10: Same as Figure 9 for the msc

The knowledge of the total mss and effective msc makes it possible to obtain the absolute level of NRCS at nadir. From equation II.20 we have:

$$
\sigma^{0}(0)=\frac{|R|^{2}}{\operatorname{mss}}\left(1+\frac{\mathrm{msc}_{e}}{8 K^{2} \mathrm{mss}^{2}}\right)
$$

Figure 11 shows the recalibration of the relative TRMM data at nadir and a comparison with the absolute values. A discrepancy from 0.5 (small winds) to $1 \mathrm{~dB}$ (larger winds) is observed, suggesting that 
the absolute values of the TRMM data might be slightly underestimated. Once recalibrated, the TRMM data are in closer agreement with the Jason $2 \mathrm{Ku}$ data, but more than $2.5 \mathrm{~dB}$ higher than the Topex data which have been shown to be already offset by $\sim 1.9 \mathrm{~dB}$ with respect to original calibration of TRMM [38].

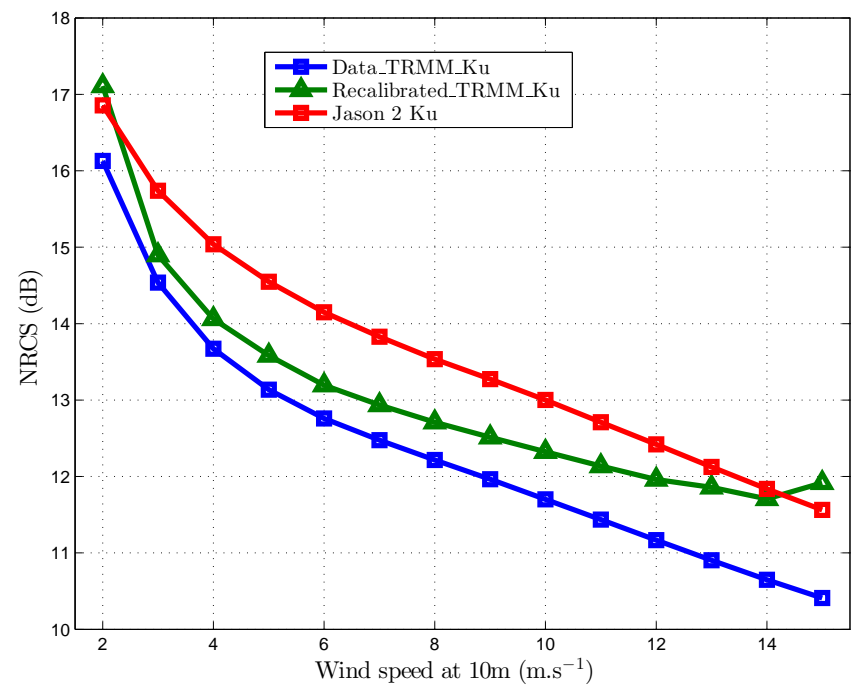

Fig. 11: Recalibration of TRMM relative data and comparison with the absolute values

\section{SRA}

The sea state conditions encountered during the SOWEX experiment (South West Tasman Sea) were found close to infinite fetch [32], [33], which makes the comparison with other satellite data and CM-mss meaningful. The relative values (i.e normalized by their maximum) of the omnidirectional NRCS recorded by the SRA were fitted in the form ([33]):

$$
\ln \left(\sigma_{r e l}^{0}\right)(\theta)=-A S^{2}+B S^{4}
$$

with $S=\tan \theta$ and $B=0.567 A^{1.332}$. The value of $A$ are not given in the publication but have been provided by one of the authors (B. Chapron). The wind speed dependence between 3 and $16 \mathrm{~m} / \mathrm{s}$ is as follows: $\mathrm{A}(3)=2.36, \mathrm{~A}(4)=46.73, \mathrm{~A}(5)=42.55, \mathrm{~A}(6)=39.37, \mathrm{~A}(7)=37.18, \mathrm{~A}(8)=34.36, \mathrm{~A}(9)=29.67, \mathrm{~A}(10)=27.17, \mathrm{~A}(11)=25.00$ $, \mathrm{A}(12)=23.30, \mathrm{~A}(13)=21.80, \mathrm{~A}(14)=20.48, \mathrm{~A}(15)=19.31, \mathrm{~A}(16)=18.27$. Figure 12 shows the estimation of the mss 
after the isotropic GO2 and GO4 models, respectively. For this estimation the first 20 degrees of the diagram have been used and error bars around the GO4 mss shows the effect of changing by \pm 2 degrees the maximum incidence angle.

As expected, the estimated mss with $\mathrm{GO} 4$ is consistent with CM-mss while the mss parameter inferred from GO2 is consistent with a radar-mss in Ka band (calculated here with help of Elfouhaily spectral model). Note, however, that the GO4-mss is found $10-20 \%$ smaller than CM-mss at moderate wind speeds. Possible explanations for this reduced slope can be hypothesized. A first artifact is the discarding of directional effects when estimating the total mss with an isotropic model. Denoting $\beta=m s s_{y} / \mathrm{mss}_{x}$ the ratio of upwind to croswind mss, it was shown in [30] that the mss estimated from the shape parameter of the GO2 model is in fact lowered by a factor $4 \beta /(1+\beta)^{2}$ with respect to the actual mss. For anisotropic wind-wave sea states such as those encountered in Cox and Munk experiment $(\beta \simeq 0.65)$ the reduction factor is negligible (0.95). However, for more pronounced anisotropy due to young sea states or the presence of a swell aligned with wind, a stronger discrepancy can be achieved. Another source of difference with CM-mss is the presence of swell which is believed to decrease the spectral density in the short-wave portion of intermediate-scale waves [25], [39]. This is consistent with the low mss observed at intermediate wind speeds $(6-10 \mathrm{~m} / \mathrm{s})$ for which run days a strong swell was reported [32].

Figure 13 shows the estimation of the non-Gaussian effective msc from the GO4 model using the first 20 degrees of the diagram and a comparison with the value predicted by the different spectra under a peakedness correction of $\lambda_{4}=0.4$. Again, error bars around the GO4 $\mathrm{msc}_{e}$ show the effect of changing by \pm 2 degrees the maximum incidence angle in the fitting process. The fluctuations with respect to the chosen angular interval are much smaller than those obtained with the TRMM, due to a higher available angular sampling of the SOWEX data. A good agreement is reached at moderate and large wind speed while the estimated effective msc is significantly lower than its predicted values at small wind speed. Again, we hypothesize that this discrepancy is due, at least partially, to the influence of swell on the short-wave spectrum. 


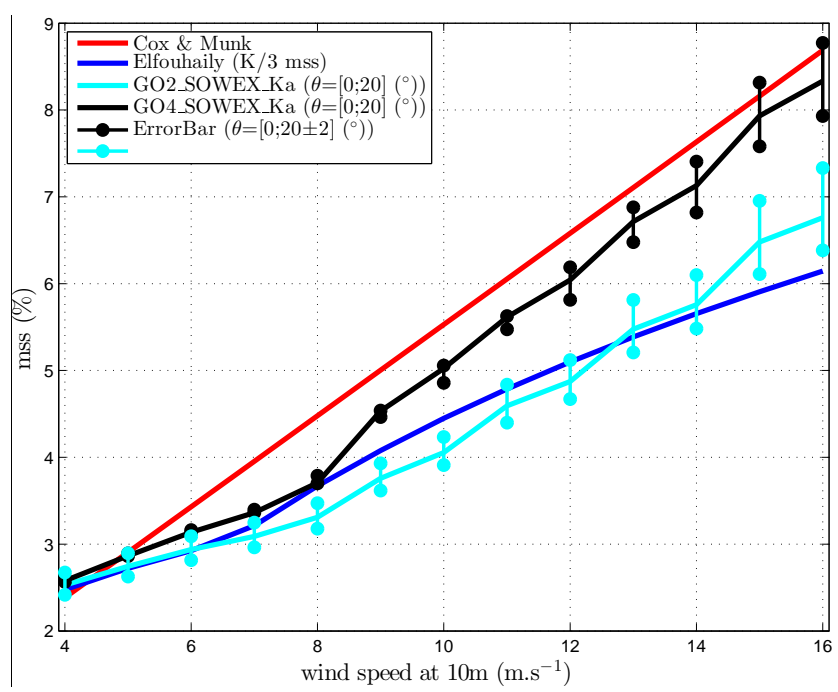

Fig. 12: mss versus wind speed from SOWEX Ka data. The error bars indicate the sensibility to a small change in the chosen angular domain for the GO2 and GO4 minimization process (VII.42).

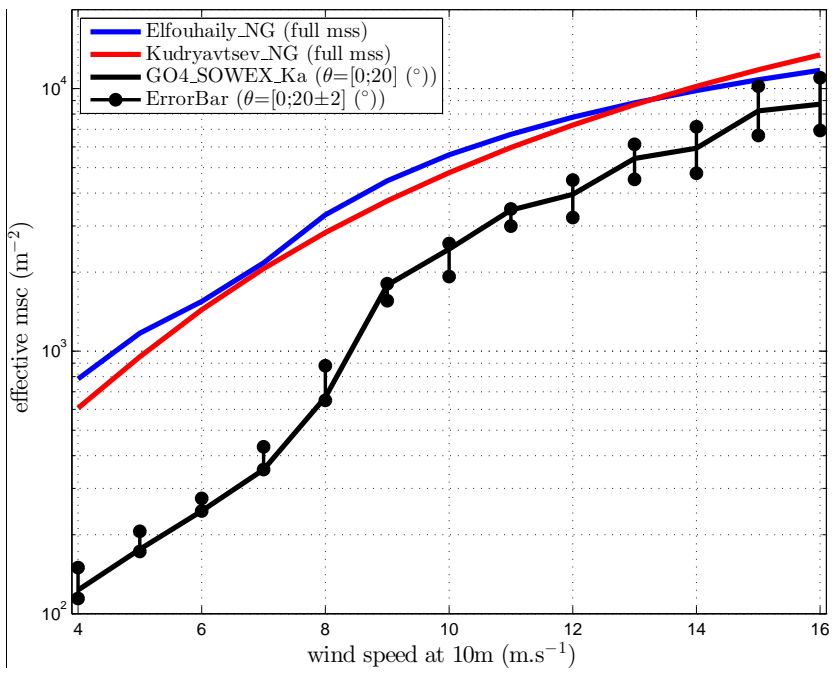

Fig. 13: msce versus wind speed from SOWEX Ka data.

\section{Jason2}

We now consider the absolute nadir NRCS of Jason 2 in C and Ku band. Figure 14 shows the mss inverted from $\mathrm{GO} 2$ at nadir in $\mathrm{Ku}$ and $\mathrm{C}$ band and a comparison with the optical mss from Cox and Munk and the radar mss according to Elfouhaily spectral model. As expected, the GO2-inverted mss is consistent with a filtered mss and much smaller than the total mss: it is found equal to about 50-60 \% of the optical mss in $\mathrm{Ku}$ band (consistently with the findings of [40]) and 35-45\% in C band.The effective msc has been evaluated using the GO4 model assuming that the mss is given by Cox and Munk measurements. 
Figure 15 shows the evolution of the non-Gaussian effective msc with wind speed for the $\mathrm{C}$ and $\mathrm{Ku}$ band of Jason 2 data. The effective msc is in good agreement with the theoretical predictions according to the different spectral models. The Ku effective msc is comparable to what was found with the TRMM data (Figure 10) based on the incidence dependence of relative NRCS.

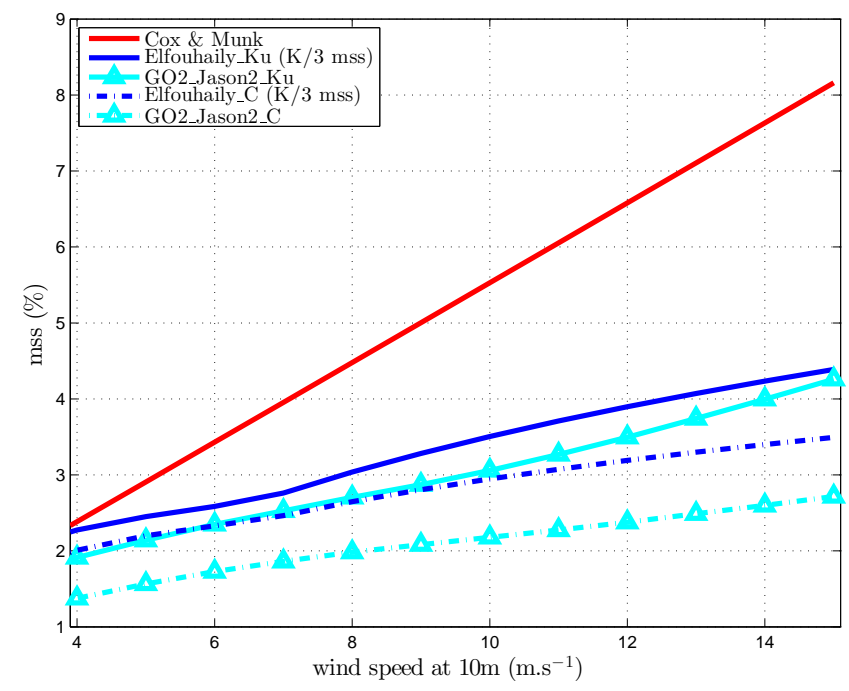

Fig. 14: mss versus wind speed from Jason $2 \mathrm{C}$ and $\mathrm{Ku}$ data.

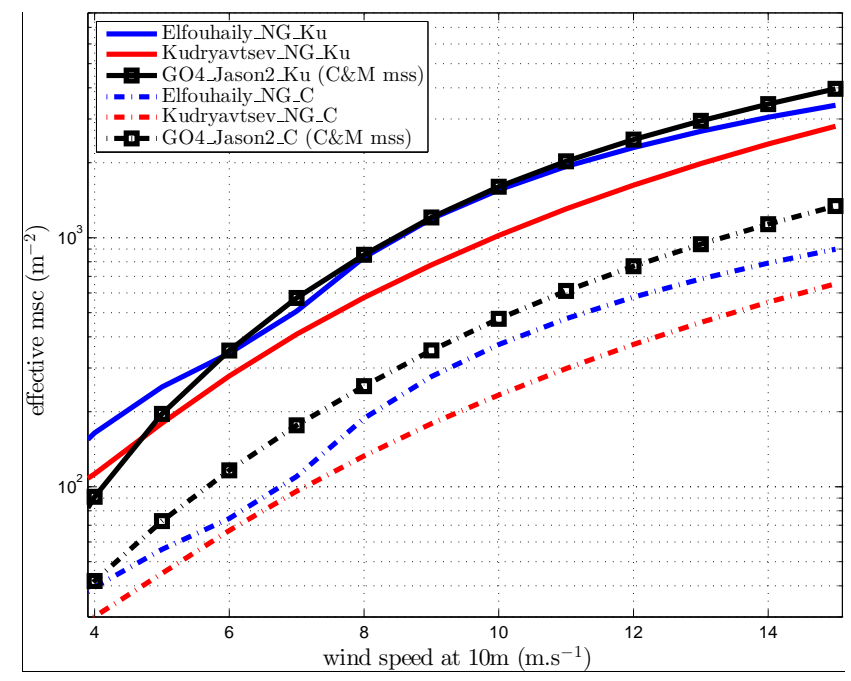

Fig. 15: msce versus wind speed from Jason2 $\mathrm{C}$ and $\mathrm{Ku}$ data.

At this point it is important to evaluate the accuracy of the effective msc when estimated from an assumed value of mss and absolute NRCS. Assuming a small variation $\Delta$ mss about a reference value of mss, we have by differentiation of (VII.43) a variation:

$$
\Delta \mathrm{msc}=24 K^{2} \mathrm{mss}^{2} \frac{\sigma^{0}}{|R|^{2}} \Delta \mathrm{mss}-16 K^{2} \mathrm{mss} \Delta \mathrm{mss}
$$


Since $\frac{\sigma^{0}}{|R|^{2}} \simeq 1 /$ mss this leads to:

$$
\frac{\Delta \mathrm{msc}}{\operatorname{msc}} \simeq \chi \frac{\Delta \mathrm{mss}}{\mathrm{mss}}
$$

where the coefficient

$$
\chi=\frac{8 K^{2} \mathrm{mss}^{2}}{\mathrm{msc}}
$$

indicates the sensibility of the relative error in msc with respect to a relative error in $\mathrm{mss}$. At $10 \mathrm{~m} / \mathrm{s}$ we have for instance $\chi \simeq 2$ in $\mathrm{Ku}$ band and $\chi \simeq 5$ in Ka band. The estimation of the effective msc can thus be considered robust to small relative errors in slope.

\section{E. Synthesis of all data sets}

There are no available near-nadir L band data to test the GO4 model. However, an approximate derivation of the L band msc can be inferred from Cox and Munk sea surface slopes measurements. In their experiment, these authors also measured the "slick" mss corresponding to the case of oil slickened surface. It is estimated that the viscous effect of surfactant damps the short-scale component smaller than about 30$40 \mathrm{~cm}$ at the sea surface. Hence, the slick mss can be seen as a radar-filtered mss at a cut-off corresponding to the L band wave number. Assuming the GO2 model with filtered mss to be close to the actual NRCS at nadir we have the following relationship with the effective $\mathrm{L}$ band msc which can thus be inverted:

$$
\frac{|R|^{2}}{\mathrm{mss}_{\text {slick }}}=\frac{|R|^{2}}{\mathrm{mss}_{\text {clean }}}\left(1+\frac{\mathrm{msc}_{e}}{8 K^{2} \mathrm{mss}_{\text {clean }}^{2}}\right)
$$

The $\mathrm{L}$ band effective msc can be extracted easily from this relation.

The airborne experiment described in [36] provides additional nadir Ka band measurements of the NRCS in ocean conditions. We have used the absoluted values of the NRCS reported in this work assuming the total mss is consistent with Cox and Munk observations to obtain the Ka band effective msc. The estimation was not conclusive at small wind speeds where negative values of the msc occured. This might be due either to a discrepancy with CM conditions or to a bias in the absolute NRCS. 
Figures 16 and 17 summarize our findings on the basis of available experimental data sets. They show, respectively, the evolution of the effective msc as a function of wind speed for the different radar bands and a function of the frequency band at different wind speeds. The effective msc is augmented by several order of magnitudes from $\mathrm{L}$ to $\mathrm{Ka}$ band and by one order of magnitude from small to large wind speeds. The cross-over observed between $\mathrm{Ku}$ and $\mathrm{Ka}$-band at small wind speed is probably due to the peculiar sea state of the SOWEX experiment and the corresponding low msc as discussed above.

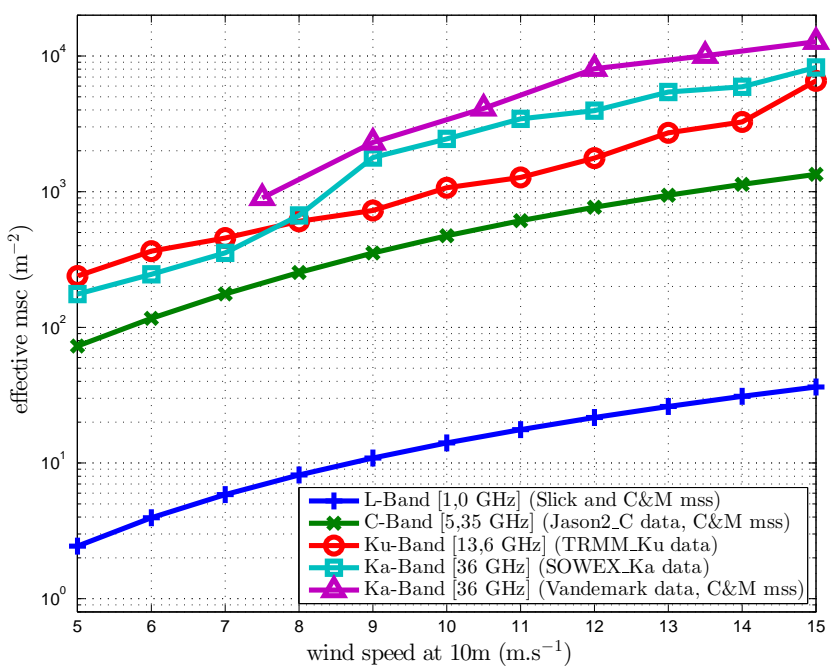

Fig. 16: Effective msc versus wind speed for the different frequency bands

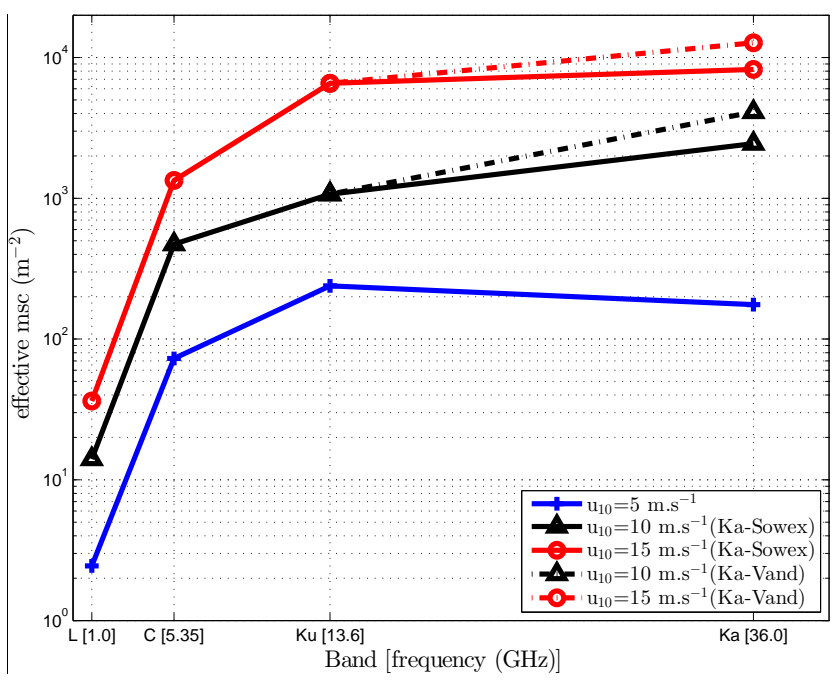

Fig. 17: Effective msc versus radar frequency at different wind speeds 


\section{CONCLUSIONS}

The GO4 is a simple scattering model with a reduced number of parameters ( 2 in the isotropic case, 5 in the directional case) which enjoys the same accuracy as the PO model in a wide range of incidence away from nadir depending on the frequency band and wind speed $(\sim 15$ degree in Ka band, $\sim 12$ degree in $\mathrm{Ku}$ band and $\sim 10$ degree in $\mathrm{C}$ band). It avoids the heuristic choice of a "radar-mss" or filtered mss used in the classical GO2 model, which is replaced by the total mss and a diffraction correction quantified by an effective msc depending on the EM frequency. While the total msc is an ill-defined quantity as it dramatically sensitive to scales much smaller than the radar wavelength and questions the microscopic nature of the sea surface, the effective msc involves only scales comparable to the radar wavelength and quantifies the diffraction process at the given wavelength. On the contrary, the total mss is a well-defined quantity as the decrease of the surface spectrum ensure convergence of its second moment. We have provided a way to calculate the effective msc from the knowledge of the surface spectrum and given estimations based on some classical spectral models as well as experimental data. Beside an accurate and simple parametrization of the scattering cross-section at moderate incidence, the GO4 is found useful in estimating the total mss (and not the radar-mss) as well as recalibrating relative data. Another interesting feature of the GO4 approach is the capability to absorb non-Gaussian effects (due to the peakedness of wave slopes and compound wave statistics) in the same analytical framework at the simple cost of an augmented msc. At this stage, the estimation process has been limited to omnidirectional quantities, the full study of the directional case being left for further work.

\section{ACKNOWLEDGMENTS}

Olivier Boisot $\mathrm{PhD}$ is granted by CNES and CLS. Frédéric Nouguier acknowledges support of the CNRS and the University of Toulon (chaire mixte). 


\section{REFERENCES}

[1] G.R. Valenzuela. Theories for the interaction of electromagnetic and oceanic waves- a review. Boundary-Layer Meteorology, 13(1):61$85,1978$.

[2] T. Elfouhaily and C.A. Guérin. A critical survey of approximate scattering wave theories from random rough surfaces. Waves in Random and Complex Media, 14(4):1-40, 2004.

[3] A. Voronovich. Small-slope approximation for electromagnetic wave scattering at a rough interface of two dielectric half-spaces. Waves in Random and Complex Media, 4(3):337-367, 1994.

[4] A. G. Voronovich. Wave scattering from rough surfaces. Springer Series on Wave Phenomena. Springer, 1994.

[5] AG Voronovich and VU Zavorotny. Theoretical model for scattering of radar signals in k u-and c-bands from a rough sea surface with breaking waves. Waves in Random Media, 11(3):247-269, 2001.

[6] AA Mouche, B. Chapron, and N. Reul. A simplified asymptotic theory for ocean surface electromagnetic wave scattering. Waves in

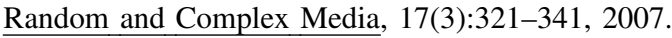

[7] G. Soriano and C.A. Guérin. A cutoff invariant two-scale model in electromagnetic scattering from sea surfaces. Geoscience and Remote Sensing Letters, IEEE, 5(2):199-203, 2008.

[8] C.A. Guérin, G. Soriano, and B. Chapron. The weighted curvature approximation in scattering from sea surfaces. Waves in Random and Complex Media, 14(3):349-363, 2010.

[9] PJ Lynch. Curvature corrections to rough-surface scattering at high frequencies. The Journal of the Acoustical Society of America, 47(3B):804-815, 1970.

[10] Ernesto Rodriguez. Beyond the kirchhoff approximation. Radio Science, 24(5):681-693, 1989.

[11] Ernesto Rodríguez. Beyond the kirchhoff approximation ii electromagnetic scattering. Radio science, 26(1):121-132, 1991.

[12] DR Thompson, TM Elfouhaily, and JL Garrison. An improved geometrical optics model for bistatic GPS scattering from the ocean surface. IEEE Transactions on Geoscience and Remote Sensing, 43(12):2810-2821, 2005.

[13] D Hauser, G Caudal, Sebastien Guimbard, and AA Mouche. A study of the slope probability density function of the ocean waves from radar observations. Journal of Geophysical Research: Oceans (1978-2012), 113(C2), 2008.

[14] AA Mouche, B. Chapron, N. Reul, D. Hauser, and Y. Quilfen. Importance of the sea surface curvature to interpret the normalized radar cross section (DOI 10.1029/2006JC004010). J. Geophys. Res., 112(C10):10002, 2007.

[15] Ninoslav Majurec, Joel T Johnson, Simone Tanelli, and Stephen L Durden. Comparison of model predictions with measurements of ku-and ka-band near-nadir normalized radar cross sections of the sea surface from the genesis and rapid intensification processes experiment. IEEE Trans. Geosci. and Remote Sens., 52(9):5320 - 5332, 2014.

[16] Suzanne T McDaniel. Microwave backscatter from non-gaussian seas. Geoscience and Remote Sensing, IEEE Transactions on, 41(1):52-58, 2003.

[17] I.M. Fuks. Backscattering from a statistically rough 2-d surface: Diffraction corrections to geometrical optics cross sections. $\underline{\text { Radio }}$ Science, 42(6):RS6S25, 2007. 


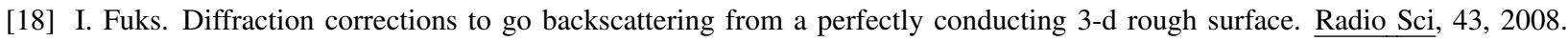

[19] Sébastien Guimbard. Interprétation et modélisation de mesures à distance de la surface marine dans le domaine micro- onde. PhD thesis, Versailles-St Quentin en Yvelines, 2010.

[20] Alexandra Bringer, Charles-Antoine Guérin, Bertrand Chapron, and Alexis Mouche. Peakedness effects in near-nadir radar observations of the sea surface. Geoscience and Remote Sensing, IEEE Transactions on, 50(9):3293-3301, 2012.

[21] T. Elfouhaily, B. Chapron, K. Katsaros, and D. Vandemark. A unified directional spectrum for long and short wind-driven waves. J. Geophys. Res., 102(C7):15781-15796, July 1997.

[22] Alexandra Bringer, Bertrand Chapron, Alexis Mouche, and Charles-Antoine Guérin. Revisiting the short-wave spectrum of the sea surface in the light of the weighted curvature approximation. IEEE Trans. Geosci. and Remote Sens.,, 52(1):679-689, 2014.

[23] V. Kudryavtsev, D. Hauser, G. Caudal, and B. Chapron. A semiempirical model of the normalized radar cross-section of the sea surface 1. background model. J. Geophys. Res, 108(C3):8054, 2003.

[24] V. Kudryavtsev, D. Hauser, G. Caudal, and B. Chapron. A semiempirical model of the normalized radar cross-section of the sea surface: 2. Radar modulation transfer function. J. Geophys. Res, 108(C3):8055, 2003.

[25] Paul A Hwang. Observations of swell influence on ocean surface roughness. Journal of Geophysical Research: Oceans (1978-2012), 113(C12), 2008.

[26] MV Yurovskaya, VA Dulov, Bertrand Chapron, and VN Kudryavtsev. Directional short wind wave spectra derived from the sea surface photography. Journal of Geophysical Research: Oceans, 118(9):4380-4394, 2013.

[27] Gary S Brown. Backscattering from a gaussian-distributed perfectly conducting rough surface. Antennas and Propagation, IEEE Transactions on, 26(3):472-482, 1978.

[28] Frédéric Nouguier. Diffraction électromagnétique par la surface océanique: influence des nonlinéarités et de lécume. PhD thesis, Aix-Marseille Université, 2009.

[29] C. Cox and W. Munk. Statistics from the sea surface derived from the sun glitter. J. Marine Res., 13:198-227, 1954.

[30] B. Chapron, V. Kerbaol, D. Vandemark, and T. Elfouhaily. Importance of peakedness in sea surface slope measurements and applications. Journal of geophysical research, 105:17, 2000.

[31] T. Elfouhaily, S. Guignard, R. Awadallah, and D.R. Thompson. Local and non-local curvature approximation: a new asymptotic theory for wave scattering. Waves Random Media, 13:321-337, 2003.

[32] Michael L Banner, Wei Chen, Edward J Walsh, Jorgen B Jensen, Sunhee Lee, and Chris Fandry. The southern ocean waves experiment. part i: Overview and mean results. Journal of physical oceanography, 29(9):2130-2145, 1999.

[33] E.J. Walsh, M.J. Banner, C.M Wrigth, D.C Vandermark, B Chapron, J Jensen, and L. See. The Southern Ocean Waves Experiment. Part III: Sea Surface Slope Statistics and Near-Nadir Remote Sensing. Journal of Physical Oceanography, 38:670-684, 2008.

[34] C. Kummerow, W. Barnes, T. Kozu, J. Shiue, and J. Simpson. The tropical rainfall measuring mission (trmm) sensor package. Journal of Atmospheric and Oceanic Technology, 15(3):809-817, 1998.

[35] N. Tran, B. Chapron, and D. Vandemark. Effect of long waves on ku-band ocean radar backscatter at low incidence angles using trmm 
and altimeter data. Geoscience and Remote Sensing Letters, IEEE, 4(4):542-546, 2007.

[36] D. Vandemark, B. Chapron, J. Sun, G.H. Crescenti, and H.C. Graber. Ocean wave slope observations using radar backscatter and laser altimeters. J. Phys. Oceanogr., 34:2825-2842, december 2004.

[37] E.J. Walsh, D.C. Vandemark, C.A. Friehe, S.P. Burns, and D. Khelif. Measuring sea surface mean square slope with a 36 GH scanning radar altimeter. J. Geophys. Res., 183(C6):12613-12602, 1998.

[38] Michael H Freilich and Barry A Vanhoff. The relationship between winds, surface roughness, and radar backscatter at low incidence angles from trmm precipitation radar measurements. Journal of Atmospheric and Oceanic Technology, 20(4):549-562, 2003.

[39] Paul A Hwang and William J Plant. An analysis of the effects of swell and surface roughness spectra on microwave backscatter from the ocean. Journal of Geophysical Research: Oceans (1978-2012), 115(C4), 2010.

[40] FC Jackson, WT Walton, DE Hines, BA Walter, and CY Peng. Sea surface mean square slope from ku-band backscatter data. Journal of Geophysical Research, 97(C7):11411-11, 1992. 